\title{
Thermo-mechanical modeling of scanning Joule expansion microscopy imaging of single-walled carbon nanotube devices
}

\author{
Song, Jizhou, jzsong@zju.edu.cn, Zhejiang University; Xie, Xu; Rogers, John,
} University of Illinois at Urbana-Champaign, United States

\begin{abstract}
An analytical model, validated by experiments and finite element simulations, is developed to study the thermal imaging of single-walled carbon nanotube (SWNT) devices by scanning Joule expansion microscopy (SJEM). A simple scaling law for thermal expansion at low frequencies, which only depends on two nondimensional geometric parameters, is established. Such a scaling law provides a simple way to determine the surface temperature distribution and power dissipation per unit length in SWNT from the measured thermal expansion in experiments. The results suggest the spatial resolution of SJEM measurement is as good as $\sim 50 \mathrm{~nm}$.
\end{abstract}

